\title{
Multimodal Intraoperative Spinal Cord Monitoring during Spinal Deformity Surgery: Efficacy, Diagnostic Characteristics, and Algorithm Development
}

\author{
Athanasios I. Tsirikos Andrew D. Duckworth Lindsay E. Henderson \\ Ciara Michaelson \\ Scottish National Spine Deformity Centre, Royal Hospital for Sick Children, Edinburgh, UK
}

\section{Highlights}

- Spinal surgery carries high risks for major neurological complications which can produce permanent functional deficits.

- We present the largest single-surgeon series of paediatric patients who underwent spinal deformity correction using consistent surgical/anaesthetic/neuromonitoring techniques.

- The multimodal technique allowed performing surgery with no neural complications; we propose an algorithm of action in response to intraoperative monitoring events.

\section{Keywords}

Spinal deformity · Scoliosis · Spinal fusion · Intraoperative spinal cord monitoring

\begin{abstract}
Objective: This study aims to present the diagnostic characteristics of multimodal intraoperative monitoring (IOM) in spinal deformity surgery and to define and categorise the neuromonitoring events, as well as propose an algorithm of action. Materials and Methods: We reviewed 1,155 consecutive patients (807 female, 348 male) who underwent deformity correction using standardised perioperative care, cortical/cervical somatosensory evoked potentials (SSEPs), and upper/lower limb transcranial electrical motor evoked potential (MEPs) by a single surgeon. The mean age at surgery was 13.8 years (range 10-23.3). We categorised IOM events
\end{abstract}

as true, transient true, and false positive or negative. Diagnostic performance criteria were calculated. Results: The most common diagnosis was adolescent idiopathic scoliosis in 717 (62\%) patients. We identified 3 true positive monitoring events occurring in 2 patients $(0.17 \%), 8$ transient true positive $(0.69 \%)$, and 8 transient false positive events $(0.69 \%)$. There were no false negative events and no patient had postoperative neurological complications. The multimodal IOM technique had a sensitivity of $100 \%$, specificity of $99.3 \%$, positive predictive value of $55.6 \%$, and negative predictive value of $100 \%$. Sensitivity was $100 \%$ for MEPs and multimodal monitoring compared to $20 \%$ for cortical or cervical SSEPs. The frequency of true or transient true positive events was higher $(p=0.07)$ in Scheuermann's kyphosis (3/91 patients, $3.3 \%$ ) compared to adolescent idiopathic scoliosis (6/717 patients, $0.84 \%$ ). Conclusion: Multimodal IOM is highly sensitive and specific for spinal cord injury. This technique is reli-

\begin{tabular}{ll}
\hline KARGER & $\begin{array}{l}\text { ( ) } 2019 \text { The Author(s) } \\
\text { Published by S. Karger AG, Basel }\end{array}$ \\
E-Mail karger@karger.com & $\begin{array}{l}\text { This is an Open Access article licensed under the Creative Commons } \\
\text { Attribution-NonCommercial-4.0 International License (CC BY-NC) } \\
\text { (http://www.karger.com/Services/OpenAccessLicense), applicable to } \\
\text { the online version of the article only. Usage and distribution for com- } \\
\text { mercial purposes requires written permission. }\end{array}$
\end{tabular}

Athanasios I. Tsirikos

Scottish National Spine Deformity Centre, Royal Hospital for Sick Children University of Edinburgh

Sciennes Road, Edinburgh EH9 1LF (UK)

E-Mail atsirikos@ hotmail.com 
able for the assessment of the condition of the spinal cord during major deformity surgery. We propose an algorithm of intraoperative action to allow close cooperation between the surgical, anaesthetic, and neurophysiology teams and to prevent neurological deficits. @2019 The Author(s)

Published by S. Karger AG, Basel
Table 1. Description of our diagnostic criteria for IOM events

\begin{tabular}{ll}
\hline $\begin{array}{l}\text { Diagnostic } \\
\text { performance } \\
\text { characteristic }\end{array}$ & Criteria \\
\hline True positive & $\begin{array}{l}\text { Lost IOM responses } \\
\text { Linked to patient positioning on the surgical } \\
\text { table or a surgical event (occurring after } \\
\text { instrumentation placement, spinal osteotomies, } \\
\text { deformity correction manoeuvres) }\end{array}$ \\
& $\begin{array}{l}\text { Responses do not return despite appropriate } \\
\text { change in patient position or surgical action } \\
\text { Potential postoperative neurological deficit }\end{array}$
\end{tabular}

\begin{tabular}{ll}
\hline Transient true & Lost IOM responses \\
positive & Linked to patient positioning on the surgical \\
& table or a surgical event (occurring after \\
& instrumentation placement, spinal osteotomies, \\
& deformity correction manoeuvres) \\
& Responses return following appropriate change \\
& in patient position or surgical action \\
& No postoperative neurological deficit
\end{tabular}

Transient false Lost IOM responses

positive Not linked to a clear surgical event (possibly associated with an anaesthetic, metabolic, technical, or unknown cause)

Responses return following appropriate action (for example increase in blood pressure, reversal of muscle relaxation, change in anaesthetic agents)

No postoperative neurological deficit tials (MEPs) [1, 4-6]. This combined approach can increase sensitivity and specificity for detecting neurological damage during deformity correction [1, 5, 7-10]. Despite the widespread use of monitoring technology, there is limited data on the efficacy of multimodal IOM in paediatric/adolescent deformity surgery [10]. The aim of our study was to present the diagnostic characteristics of multimodal IOM in young patients undergoing deformity correction, to define the types of neuromonitoring changes in relevance to surgical events, and to propose an algorithm of action.

\section{Materials and Methods}

We included 1,155 consecutive patients (807 females, 348 males) with a mean age at surgery of 13.8 years (range 10-23.3). All patients underwent spinal deformity surgery with instrumentation and multimodal IOM under the care of the senior author between 2009 and 2017. We reviewed the medical, surgical, and IOM records and documented postoperative complications.

Anaesthetic Technique

We premedicated our patients with midazolam $(0.5 \mathrm{mg} / \mathrm{kg}$ to maximum $20 \mathrm{mg})$ and clonidine $(1 \mu \mathrm{g} / \mathrm{kg})$. We used a total intravenous anaesthesia technique. Induction was done with pro-

IOM Preventing Neurological Injury in

Spinal Deformity Surgery
Med Princ Pract 2020;29:6-17

pofol TCi and bolus of atracurium $(0.5 \mathrm{mg} / \mathrm{kg})$ to facilitate intubation. The Paedfusor pharmacokinetic model was used for children below 16 years, and the Marsh model for those 16 years and older. Anaesthesia was maintained with infusion of propofol and remifentanil, and ventilation with an air/oxygen mixture. A bispectral index monitor was used to optimise the depth of anaesthesia. An arterial line was inserted in all patients and a central venous line where clinically indicated. Once baseline IOM traces were obtained, another dose of atracurium up to $0.5 \mathrm{mg} /$ $\mathrm{kg}$ was given during surgical dissection. At this time, the blood pressure was controlled to a minimum mean of $55 \mathrm{~mm} \mathrm{Hg}$. This was achieved by titrating the remifentanil infusion. At the time of placement of instrumentation and during deformity correction, the blood pressure was raised to a minimum mean of 65 $\mathrm{mm} \mathrm{Hg}$. Following the last IOM check and during wound closure, propofol was discontinued and desflurane was commenced to achieve rapid patient wake up at the conclusion of surgery. Cell salvage was routinely used to reduce allogenic blood transfusion. 


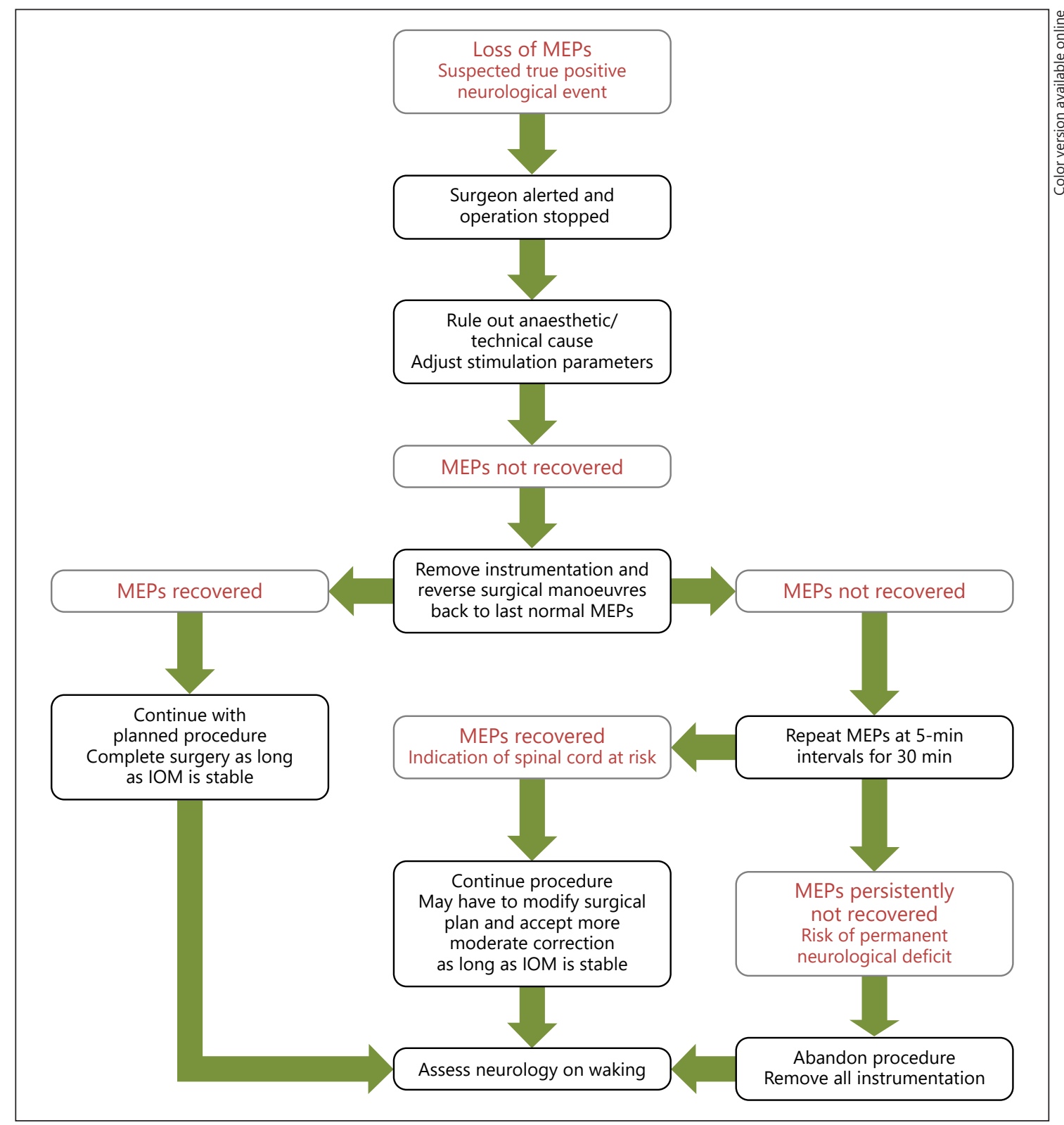

Fig. 1. Decision algorithm in response to MEP events during spinal deformity surgery.

\section{IOM Technique}

IOM was performed by 2 nationally certified senior clinical physiologists who were present throughout the entire procedure using a 10-channel Medelec ${ }^{\circledR}$ Synergy IOM constant voltage system (2009-2013) and a 24-channel Inomed ISIS IOM constant current system (2014-2017). All patients underwent multimodal monitoring, recording of cortical SSEPs, cervical SSEPs, and MEPs. Monitoring commenced after patient positioning and continued until 20 min after spinal instrumentation was complete. Reference traces were obtained before skin incision.
Somatosensory Evoked Potentials

Sensory evoked potentials were recorded from the brain (cortical SSEP) and spinal cord (cervical SSEP). Responses were evoked by cathodal delivery of $0.2 \mathrm{~ms}$ duration, square-wave impulses at a range of $3.7-4.3 \mathrm{~Hz}$ to bilateral posterior tibial nerves using pairs of adhesive electrodes. The stimulation intensity was $45 \mathrm{~mA}$. Upper limb SSEPs were introduced in a similar fashion, with square wave impulses delivered to bilateral ulnar nerves at $25 \mathrm{~mA}$. Cortical SSEPs were recorded from corkscrew electrodes placed in the scalp at $\mathrm{Cz}$ ' and referred to $\mathrm{Fz}$ (identified using the 10-20 interna- 
Table 2. IOM event characteristics if only one of the individual monitoring modalities was used against our combined multimodal technique in our cohort of 1,155 patients

\begin{tabular}{lllll}
\hline & $\begin{array}{l}\text { True and } \\
\text { transient true } \\
\text { positive }\end{array}$ & $\begin{array}{l}\text { Transient } \\
\text { false } \\
\text { positive }\end{array}$ & $\begin{array}{l}\text { False } \\
\text { negative }\end{array}$ & $\begin{array}{l}\text { True } \\
\text { negative }\end{array}$ \\
\hline Cervical SSEPs & 2 & 1 & 9 & 1,143 \\
Cortical SSEPs & 2 & 3 & 9 & 1,141 \\
MEPs & 10 & 5 & 0 & 1,140 \\
Combined & 10 & 8 & 0 & 1,137 \\
\hline
\end{tabular}

tional head measurement system). Cervical SSEPs were recorded from a needle electrode placed subcutaneously over Cv2 and referred to $\mathrm{Fz}$.

\section{Motor Evoked Potentials}

MEPs were recorded bilaterally as compound muscle action potentials (CMAPs) from the tibialis anterior (TA) and abductor hallucis $(\mathrm{AH})$ muscles in the lower extremities (rectus femoris [RF] was added in 2013) and from the abductor digiti minimi (ADM) muscle in the upper extremities. Bilateral ADM responses were used as controls to differentiate between surgical and nonsurgical loss of limb responses and identify brachial plexus compromise due to patient positioning. MEPs were elicited periodically by delivering an anodal pulse train of 4-7 pulses of $0.5 \mathrm{~ms}$ duration and interstimulus interval of $2-4 \mathrm{~ms}$ at amplitudes of 200-500 V (fixed voltage system) or 150-220 mA (fixed current system) between two corkscrew electrodes positioned over the motor cortex at C1 and C2. The stimulus strength was optimised to elicit reproducible responses. In patients with grade IV and V spondylolisthesis included in this series we added MEP recording from the anal sphincter and the gastrocnemius to the multimodal technique in order to monitor the sacral nerve roots.

\section{Primary Outcome Measure}

Our diagnostic criteria for IOM monitoring are presented in Table 1. Decisions during surgery in response to IOM findings rested with the surgeon following an algorithm that we developed (Fig. 1). Close collaboration with our anaesthetic and neurophysiology teams was mandatory to secure accurate interpretation of IOM changes, to initiate an immediate response, and prevent neurological damage. In all cases of IOM events, the routine initial check included mean arterial blood pressure that was raised to $>80 \mathrm{~mm} \mathrm{Hg}$ and body temperature $>36.5^{\circ} \mathrm{C}$. Technical problems with the recording system or due to dislodgement of the electrodes were also excluded. Muscle relaxant (atracurium) given during soft tissue dissection had metabolised and another baseline IOM recording was obtained before the placement of instrumentation.

\section{Alarm Criteria}

Action was taken when:

- Mode 1-cortical SSEPs: unilateral or bilateral responses dropped $>50 \%$ in amplitude with or without a corresponding increase in latency $>10 \%$ of reference traces.

IOM Preventing Neurological Injury in

Spinal Deformity Surgery
- Mode 2-cervical SSEPs: unilateral or bilateral responses dropped $>50 \%$ in amplitude with or without corresponding increase in latency $>10 \%$ of reference traces.

- Mode 3-MEPs: MEP-evoked CMAP responses in any muscle were lost and remained absent. Any sudden drop in amplitude of MEP traces $>80 \%$ of reference traces was reported and investigated.

\section{Statistical Methods}

SPSS version 17.0 (SPSS, Chicago, IL, USA) was used. Categorical binary data were analysed using the $\chi^{2}$ test $(n>5$, all cells) or Fisher exact test ( $n \leq 5$, at least one cell). Significance was determined as $p<0.05$.

\section{Results}

Diagnoses included adolescent idiopathic scoliosis (AIS; 717 patients, 62.0\%), syndromic scoliosis (127, $11.0 \%)$, Scheuermann's kyphosis (91, 7.9\%), congenital scoliosis $(85,7.4 \%)$, scoliosis with intraspinal anomalies $(59,5.1 \%)$, juvenile idiopathic scoliosis $(23,2.0 \%)$, infantile idiopathic scoliosis $(18,1.6 \%)$, scoliosis with congenital cardiac disease $(16,1.4 \%)$, grade IV and V lumbosacral spondylolisthesis $(15,1.3 \%)$, and deformity associated with spinal tumour $(4,0.3 \%)$. In total, 1,137 patients (98.4\%) underwent posterior, $15(1.3 \%)$ combined antero-posterior, and $3(0.3 \%)$ anterior deformity correction.

Patients with an AIS had mean upper thoracic scoliosis of $45^{\circ}$ (range $25-89^{\circ}$ ) corrected by a mean of $69 \%$, mean mid-thoracic scoliosis of $72^{\circ}$ (range 55-115 ${ }^{\circ}$ ) corrected by a mean of $72 \%$, and mean lumbar scoliosis of $62^{\circ}$ (range $48-98^{\circ}$ ) corrected by a mean of $74 \%$. Segmental pedicle screw or hybrid (proximal pedicle hooks/sublaminar wires-distal pedicle screws) instrumentation was used in patients with an AIS. Patients with Scheuermann's condition had a mean kyphosis of $96^{\circ}$ (range $85-130^{\circ}$ ) corrected to a mean of $46^{\circ}$ (range $40-62^{\circ}$ ). Posterior closing wedge osteotomies were performed in all patients and the correction was conducted using hybrid instrumentation supplemented by the iliac crest bone.

None of our patients with scoliosis or kyphosis had preoperative neural deficits and their neurological examination was normal. All 15 patients with high-grade lumbosacral spondylolisthesis had reduced sensation with pins/needles/tingling of L5/S1 distribution. They underwent posterior spinal fusion of L4-S1 with the use of bilateral L4 pedicle screws and S1 into L5 transfixation screws supplemented by the iliac crest bone. Neural decompression was not performed on any patient. Iliac screws were used in 1 patient to provide additional distal 


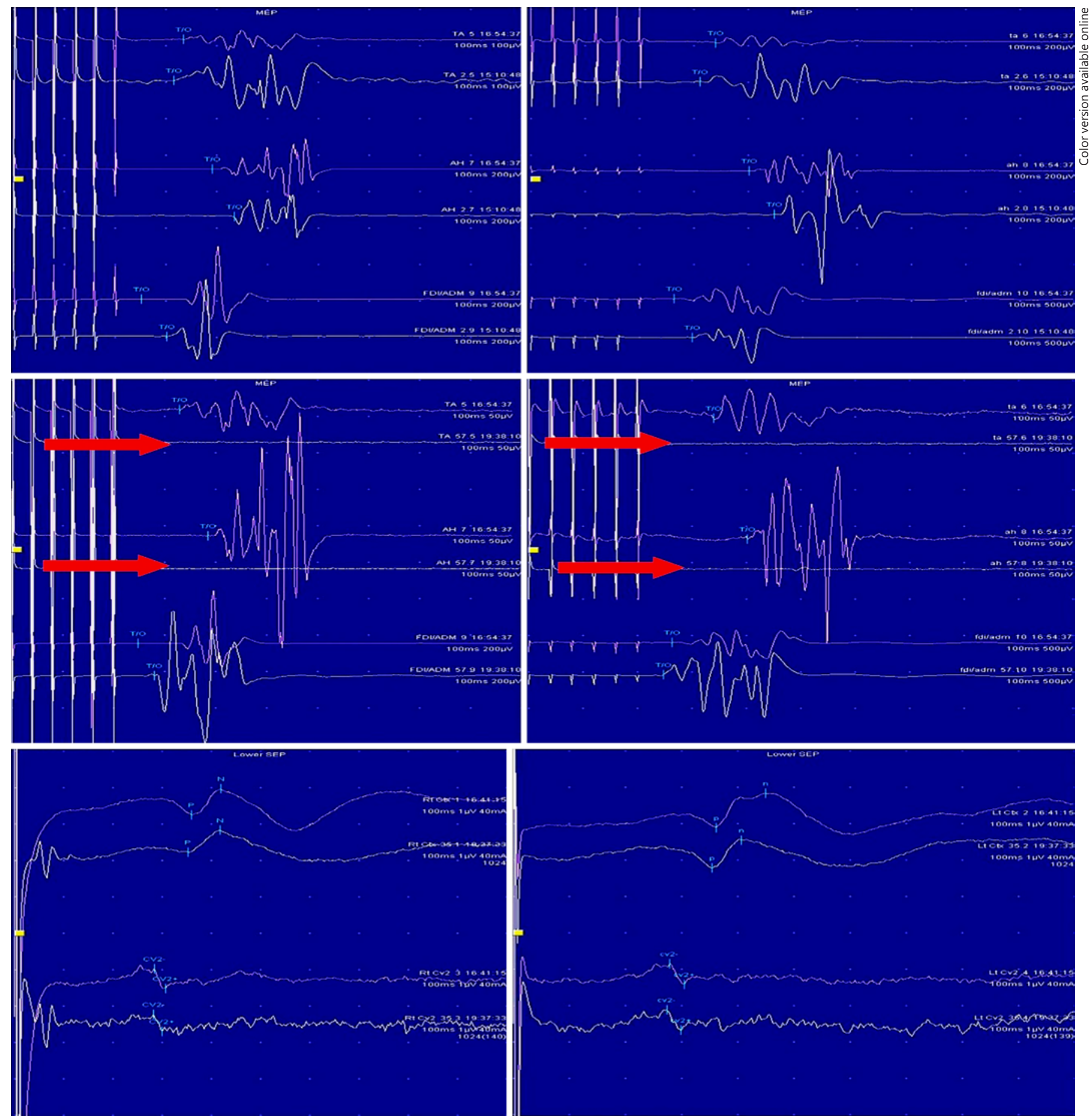

Fig. 2. A true positive event with lower limb MEPs lost during AIS posterior correction. Baseline MEP traces were present (first row). During scoliosis correction the lower limb MEPs were lost bilaterally with present MEP responses in the upper limbs (second row, arrows). Despite removing the rods and a surgical pause, the MEP traces did not return. During the time of the loss of bilateral lower limb MEPs the sensory traces remained unchanged (third row). After removal of all instrumentation, the patient woke up with no neurological deficit. The procedure was concluded 1 week later with no further problems and a good deformity correction. 
stability of the lumbosacral junction. The spondylolisthesis was improved by one grade through patient positioning on the surgical table. The patient reported neurological symptoms improved or resolved after surgery.

\section{IOM Events}

We recorded 3 true positive monitoring events occurring in 2 patients $(0.17 \%), 8$ transient true positive events $(0.69 \%)$ of which 2 were during patient positioning on the surgical table, and 8 transient false positive events (0.69\%). There were no false negative IOM events and no patient had postoperative neurological deficits (Table 2).

The first 2 true positive events occurred during deformity correction on a patient with thoracic and lumbar AIS measuring 80 and $75^{\circ}$, respectively, who underwent a posterior spinal fusion with all pedicle screw instrumentation. During the index and first revision procedure we recorded loss of MEPs while the SSEPs were present throughout (Fig. 2). As the IOM responses did not return despite the removal of instrumentation and pedicle screws, we followed our algorithm and surgery was abandoned on both occasions. The patient was neurologically normal and the postoperative spinal MRI showed no abnormalities. The surgery was concluded as planned 1 week later (2nd revision procedure) with satisfactory scoliosis correction to 22 and $20^{\circ}$ for the thoracic and lumbar curves and a good clinical outcome. The other true positive event occurred during posterior osteotomies on a diabetic patient with thoracic Scheuermann's kyphosis measuring $100^{\circ}$ consisting of the loss of MEPs and cortical and cervical SSEPs bilaterally. The IOM responses did not return despite a prolonged surgical pause and following our algorithm the surgery was abandoned. The patient had normal neurological function and postoperative spinal MRI. A revision procedure was performed 1 week later and achieved excellent kyphosis correction to $45^{\circ}$ and a good clinical outcome.

Transient true positive events occurred during posterior osteotomies in 2 patients with Scheuermann's kyphosis consisting of reduction in MEPs while the SSEPs were present throughout (Fig. 3). Following a surgical pause of $20 \mathrm{~min}$, the MEPs returned to baseline and the procedure was concluded in both patients without further problems. One patient with a thoracic AIS measuring $130^{\circ}$ underwent combined anterior spinal release $(8$ discectomies) and posterior spinal fusion with a hybrid construct performing apical translation using concave sublaminar wires. At that stage we recorded loss of bilateral MEPs which returned 10 min after release of the sublaminar wires and removal of the concave rod while the SSEPs were present throughout. A lesser degree of scolio-

IOM Preventing Neurological Injury in Spinal Deformity Surgery sis correction was performed and the procedure was concluded with stable neuromonitoring and a good outcome. A patient with $95^{\circ}$ thoracic AIS underwent posterior scoliosis correction with the use of an all-pedicle screw construct. During placement of a concave thoracic apical pedicle screw at T8, unilateral loss of lower limb MEPs was noted due to medial pedicle cortical breach with present SSEPs throughout. Following a surgical pause, the MEPs returned to baseline and the procedure was concluded without instrumenting the concave T8 level. One patient with thoracic AIS $\left(55^{\circ}\right)$ and lumbar AIS $\left(90^{\circ}\right)$ underwent a posterior spinal fusion with the use of all-pedicle screw instrumentation. During placement of a concave lumbar apical pedicle screw at L1, unilateral loss of lower limb MEPs was recorded due to medial pedicle cortical breach while the SSEPs were present throughout. After a surgical pause, the MEPs returned to baseline and the procedure was concluded without instrumenting the concave L1 level. One patient with very severe thoracic AIS $\left(110^{\circ}\right)$ underwent a posterior spinal fusion with the use of all-pedicle screw instrumentation. Posterior closing wedge osteotomies were performed to increase the flexibility of the spine and allow optimum correction. During the osteotomies, unilateral loss of lower limb MEPs was recorded while theSSEPs were present throughout. Following a surgical pause of $10 \mathrm{~min}$, the IOM traces returned to baseline and the surgery was concluded following our algorithm with no further problems.

Two additional transient true positive events occurred during patient positioning. The first was during hip extension to increase anterior sacral inclination and to allow a partial reduction of grade IV (to III) lumbosacral spondylolisthesis and consisted of unilateral loss of lower limb MEPs and cortical and cervical SSEPs. The second event occurred when 1 patient'sleft arm was overstretched beyond $90^{\circ}$ resulting in loss of left upper limb MEPs while all other modalities were present. Correction of patient position on the surgical frame resulted in the return of normal IOM in both patients and surgery proceeded to a successful outcome.

True and transient true positive events in AIS $(n=8)$ included isolated loss of MEPs with normal SSEPs throughout. True and transient true positive events in Scheuermann's kyphosis included 2 patients with isolated loss of MEPs with normal SSEPs throughout and 1 patient with loss of MEPs and cortical and cervical SSEPs.

Transient false positive events were related to low mean arterial blood pressure ( $<60 \mathrm{~mm} \mathrm{Hg}$ ) and occurred in 7 patients with AIS (Fig. 4) and 1 with Scheuermann's kyphosis, including 5 patients who had reduction in 

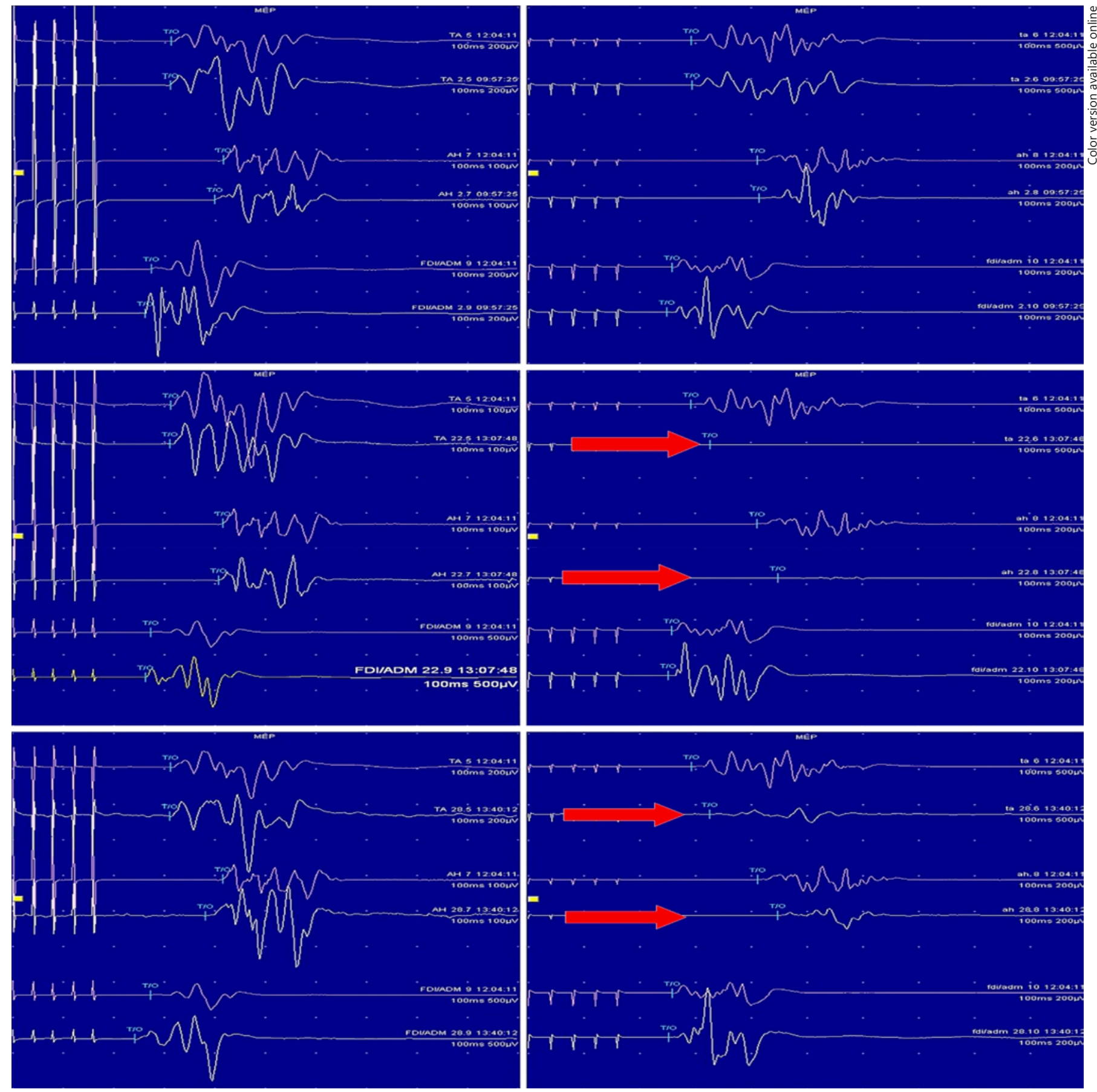

Fig. 3. A transient true positive event with unilateral loss of lower limb MEPs during posterior closing wedge osteotomies for thoracic Scheuermann's kyphosis. Baseline MEP traces were present (first row). During the posterior osteotomies the left-sided lower limb MEPs disappeared (arrows) while these were stable on the right side and in the upper limbs (second row). After $20 \mathrm{~min}$ of surgical pause, the MEPs started to return and remained stable throughout the procedure (third row, arrows). This allowed continuation of the surgery and correction of the kyphosis as initially planned. 


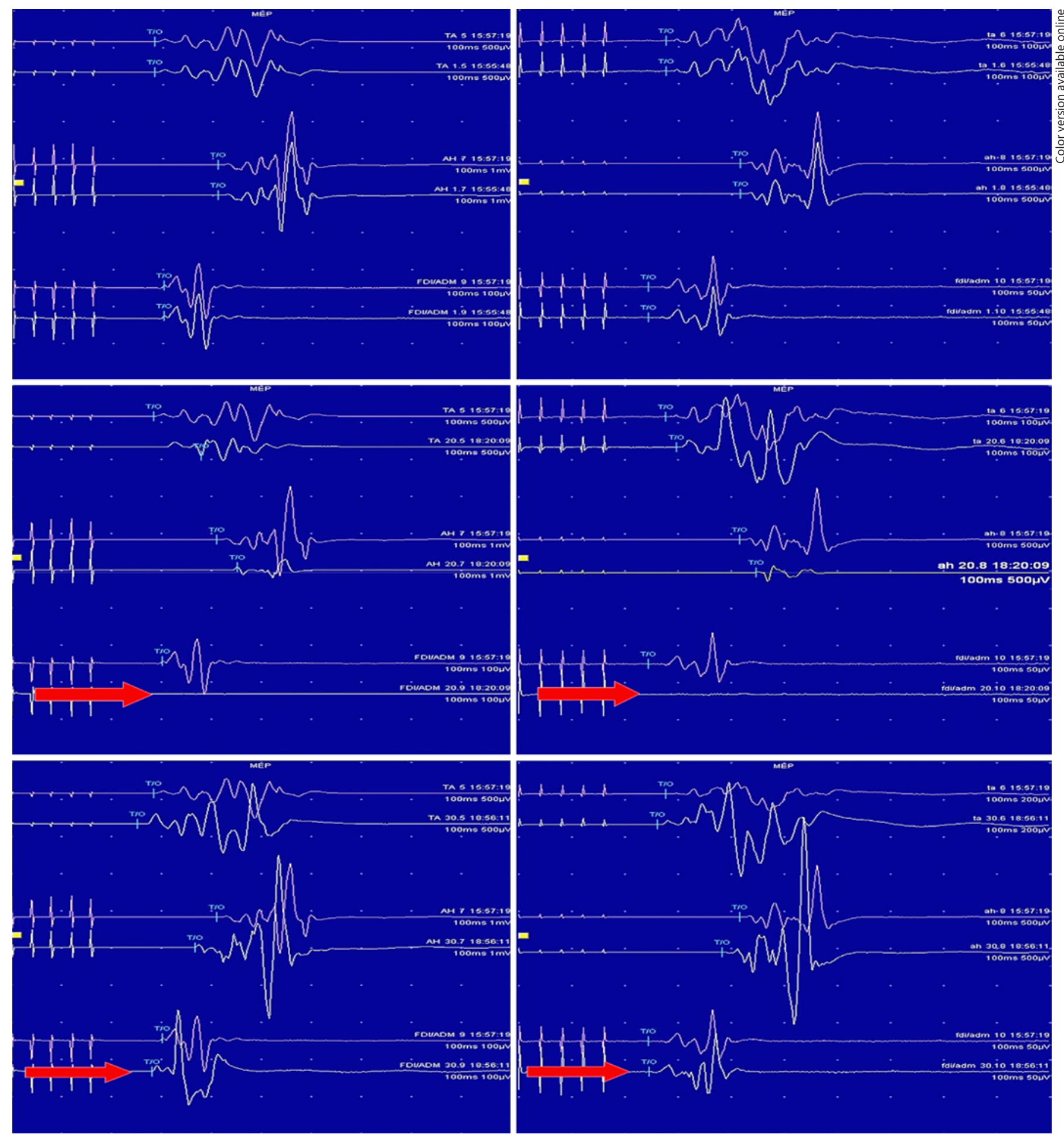

Fig. 4. A transient false positive event with bilateral loss of upper limb MEPs during AIS correction. Baseline MEP traces were present (first row). During surgery the upper limb MEPs disappeared bilaterally (arrows) with mean blood pressure $48 \mathrm{~mm} \mathrm{Hg}$ (second row). After $10 \mathrm{~min}$ of surgical pause and by raising the mean blood pressure to $68 \mathrm{~mm} \mathrm{Hg}$, the upper limb MEPs fully recovered and remained stable throughout the procedure (third row, arrows). This allowed continuation of the surgery and correction of the scoliosis as initially planned. 
Table 3. True positive events during IOM monitoring in our 1,155 patients presented according to underlying diagnosis

\begin{tabular}{llll}
\hline Diagnosis (patients, $n)$ & $\begin{array}{l}\text { Transient } \\
\text { true positive, } n\end{array}$ & $\begin{array}{l}\text { True } \\
\text { positive, } n\end{array}$ & $\begin{array}{l}\text { True positive } \\
\text { IOM rate, } \%\end{array}$ \\
\hline AIS $(n=717)$ & 5 & 1 & 0.84 \\
Syndromic scoliosis $(n=127)$ & 0 & 0 & 0 \\
Scheuermann's kyphosis $(n=91)$ & 2 & 1 & 3.3 \\
Congenital scoliosis $(n=85)$ & 0 & 0 & 0 \\
Scoliosis with intraspinal anomaly $(n=59)$ & 0 & 0 & 0 \\
Juvenile scoliosis $(n=23)$ & 0 & 0 & 0 \\
Scoliosis with congenital cardiac disease $(n=16)$ & 0 & 0 & 0 \\
Infantile scoliosis $(n=18)$ & 0 & 0 & 0 \\
Grade IV and V lumbosacral spondylolisthesis $(n=15)$ & 1 & 0 & 6.7 \\
Deformity associated with spinal tumour $(n=4)$ & 0 & 0 & 0 \\
\hline
\end{tabular}

Table 4. Diagnostic performance characteristics of IOM monitoring in our 1,155 patients

\begin{tabular}{|c|c|c|c|c|}
\hline & $\begin{array}{l}\text { Cervical SSEPs } \\
(95 \% \mathrm{CI})\end{array}$ & $\begin{array}{l}\text { Cortical SSEPs } \\
(95 \% \mathrm{CI})\end{array}$ & $\begin{array}{l}\text { MEPs } \\
(95 \% \text { CI })\end{array}$ & $\begin{array}{l}\text { Multimodal } \\
\text { technique } \\
(95 \% \mathrm{CI})\end{array}$ \\
\hline Specificity, \% & $99.91(99.51-100)$ & $99.74(99.24-99.95)$ & $99.56(98.98-99.86)$ & $99.30(98.63-99.70)$ \\
\hline Positive predictive value, $\%$ & $66.67(16.45-95.31)$ & $40.00(11.08-78.11)$ & $66.67(45.48-82.75)$ & $55.56(38.52-71.38)$ \\
\hline Negative predictive value, $\%$ & $99.31(99.06-99.49)$ & 99.30 (99.05-99.49) & $100(-)$ & $100(-)$ \\
\hline Negative likelihood ratio & $0.80(0.59-1.09)$ & $0.80(0.59-1.09)$ & $0.00(-)$ & $0.00(-)$ \\
\hline
\end{tabular}

CI, confidence interval.

MEPs, 2 who had reduction in the amplitude of cortical SSEPs, and 1 who had reduction in the amplitude of cortical/cervical SSEPs). By increasing the blood pressure ( $>80 \mathrm{~mm} \mathrm{Hg}$ ), the IOM traces returned to baseline after a surgical pause of 10-15 min and the procedures were concluded with no change in surgical planning. All 8 transient false positive events were recorded in the first 2 years of our series. As experience and coordination improved between the surgical/anaesthetic/neurophysiology teams such events were eliminated.

\section{Risk Factors for True IOM Events}

We found a difference between Scheuermann's kyphosis and AIS on the risk of true or transient true positive events with event incidence of $3 / 91$ patients $(3.3 \%)$ in Scheuermann's kyphosis versus $6 / 717$ patients $(0.84 \%)$ in AIS (Table 3), although this did not reach statistical significance ( $p=0.07$, Fisher exact test). Differences were observed between surgical approaches on the risk of true or transient true positive events with an event incidence of $10 / 1,137$ patients $(0.88 \%, 3$ true positive and 7 transient true positive) during posterior surgery versus $1 / 15 \mathrm{pa}-$ tients $(6.7 \%, 1$ transient true positive) during combined anterior/posterior spinal fusion. However, this did not reach statistical significance ( $p=0.135$, Fisher exact test).

Patients with congenital scoliosis who underwent posterior hemivertebra resection followed by instrumented fusion had no IOM events. In this group, we have managed to acquire reproducible IOM (including MEPs) in patients as young as 2.5 years. There were, however, young children below the age of 3 years in whom we performed posterior hemivertebra resection and where MEPs were not reproducible; cervical SSEP monitoring was used in these patients who were excluded from the present study. There were no IOM events during surgery among the patients with high-grade spondylolisthesis (one transient true positive event occurred during patient positioning as described previously).
Tsirikos/Duckworth/Henderson/ Michaelson 


\section{IOM Diagnostic Performance Characteristics}

The sensitivity of our multimodal IOM technique was $100 \%$ (all patients with impending spinal cord injury have a positive IOM event) and the specificity was $99.3 \%$ (patients with normal IOM had a $99.3 \%$ chance that their cord was safe). The positive predictive value was $55.56 \%$ (55.56\% chance that an IOM event reflected a surgicalrelated cause of cord injury) and negative predictive value was $100 \%$ (100\% chance that normal IOM corresponded to no cord injury; Table 4 ).

\section{Discussion}

The risk of neurological complications during deformity correction ranges from 1 to $2 \%$ [2-4]. IOM is recommended in procedures where there is risk of neurological injury including scoliosis surgery $[4,11]$. Nuwer et al. [6] published a multicentre survey reporting significant reduction in neurological injuries following scoliosis surgery due to SSEP monitoring. The ability of IOM to detect such complications has improved, predominantly as multimodal monitoring techniques have been introduced. Fehlings et al. [12], in their systematic review of 32 studies, determined that strong evidence exists that multimodal IOM is sensitive and specific for detecting intraoperative neurological injury and recommended the use of such monitoring for spinal deformity correction.

Combined IOM techniques recording SSEPs and MEPs have good diagnostic characteristics for detecting neurological injury during deformity surgery $[1,5,7-10,13]$. However, previous studies have questioned the efficacy of IOM, with reported sensitivity as low as 43\% [14]. Other authors suggested that important changes in IOM leading to permanent neurological deficits occurred between 16 and $40 \%$ of the time $[15,16]$. The evidence for the use of IOM in paediatric deformity surgery is growing due to increased risk of neurological damage during curve correction [14]. Our series shows that multimodal IOM used in conjunction with a standardised management algorithm is highly sensitive (100\%) and specific (99.3\%) in the detection of spinal cord injury. The total intravenous anaesthesia technique used in our patients can reduce the rate of false positive and transient events during corrective surgery due to dose-dependent effects on evoked potentials $[4,17-21]$. We conclude that the multimodal IOM technique is a fast and reliable method to assess the condition of the cord during deformity surgery. This immediate alert of impending neurological damage allows for early action to be taken at a potentially reversible point.

IOM Preventing Neurological Injury in Spinal Deformity Surgery
Thuet et al. [10] presented a 23-year retrospective series of 3,436 paediatric patients undergoing spinal surgery using varying IOM methods and reported a false negative rate of $0.2 \%$. This is contrary to the negative predictive value of our IOM technique which was $100 \%$ (normal IOM corresponded to no cord injury). Although MEPs had the strongest diagnostic performance characteristics in our series, we recommend the combined SSEP/ MEP monitoring technique given that an isolated intraoperative injury to the posterior cord would only be detected through loss of SSEPs.

The development of neurological complications during deformity correction can occur due to mechanical or vascular insults to the cord. An increased risk has been associated with certain types of deformity, presence of preoperative neurological deficits, combined procedures, increased blood loss, and revision surgery [2-4, 14]. Our study demonstrated an increased rate of true or transient true positive IOM events with combined anterior/posterior surgery, although this was not statistically significant due to the small number of patients included in our series (1.3\%). Scheuermann's kyphosis, where posterior closing wedge osteotomies were performed, carried increased risk of true or transient true positive events compared to AIS. Previous studies suggested greater risk of neurological complications in patients with thoracic hyper-kyphosis and those requiring corrective osteotomies [4, 22, 23], which is consistent with our findings.

The primary strength of our study is that it presents the largest single surgeon's consecutive series of young patients with severe deformities who underwent surgical correction in a single institution. We analysed a prospective database and reported the efficacy of multimodal IOM in detecting neurological injury. Our IOM protocol was standardised throughout the study period in accordance with the guidelines proposed by the British Society of Clinical Neurophysiology and the Association of Neurophysiological Scientists [24]. A consistent anaesthetic/ perioperative care pathway was used in collaboration with dedicated spinal anaesthetists.

There is no standardised definition and classification of IOM events to date. We used the alarm criteria previously described to take action and reverse our neuromonitoring events. We defined the types of IOM events in relation to surgical steps. We categorised as true or transient true positive events those occurring in relation to patient positioning or following instrumentation placement, deformity correction manoeuvres, and spinal osteotomies in order to determine necessary actions and prevent neurological damage. True positive IOM events 
where the responses do not return to baseline have the potential for postoperative neurological deficits. We did not record any false positive events in our series where the responses did not return to baseline with anaesthetic actions (for example, raise blood pressure and body temperature, reverse muscle relaxant, and adjust anaesthetic agents). We defined transient false positive events as those not linked to a clear surgical step and we felt that these events could be associated with anaesthetic, metabolic, or technical problems occurring during surgery. All transient false positive events were recorded early in our series and reversed with the responses returning to baseline when the patient's blood pressure was raised following appropriate anaesthetic actions. The relatively high number of transient false positive events ( $42 \%$ of our total IOM events) is the reason the positive predictive value is $55.56 \%$ in our series; however, such events have not occurred after the first 2 years and as the experience of our surgical/anaesthetic/neurophysiology teams increased.

We have proposed an algorithm to allow the surgical team to decide appropriate actions in response to MEP loss (Fig. 1). There are existing guidelines, checklists, and algorithms for the prevention, identification, and management of neurological complications in spine surgery $[1,12,25-29]$. We advocate the need for close cooperation, and continuous communication of the surgical/anaesthetic/neurophysiology teams before, during, and after surgery cannot be overemphasised. In our experience, this coordinated approach has allowed appropriate response to IOM changes and ensured that no patient in this series developed neurological deficits contrary to previous studies which reported risk of permanent neurological injury of $0.1-0.2 \%[23,29]$. In our patient consent, we have included for a detailed discussion on IOM and the possible need to abandon surgery depending on IOM findings and complete it in a staged manner at a later date.
We acknowledge that a limitation of the study is the fact that we have not included older adult patients with de novo or degenerative scoliosis who would be likely to require complex surgery with increased risk for IOM events and neurological complications. This group of patients are not seen in our Centre, which is commissioned to treat children, adolescents, and young adults with spinal deformities. However, within the paediatric group we have included patients who underwent posterior spinal osteotomies such as in Scheuermann's kyphosis, as well as complete hemivertebra resections in children with congenital spinal deformity. The distribution of patients presented in our series is consistent with the literature and representative of day-to-day practice in most spinal deformity centres [29].

\section{Conclusion}

Multimodal monitoring has demonstrated high sensitivity and specificity with $100 \%$ negative predictive value for detecting intraoperative spinal cord injury. This technique is safe, reproducible, and reliable for the assessment of neurological function and can be considered the current gold standard to prevent neurological complications during spinal deformity surgery.

\section{Statement of Ethics}

Ethical approval for this study was obtained from the Hospital's Research and Ethical Board.

\section{Disclosure Statement}

The authors have nothing to disclose. No benefit in any form has been obtained by a profit or non-profit organisation in relation to this study or paper.

\section{References}

1 Vitale MG, Skaggs DL, Pace GI, Wright ML, Matsumoto H, Anderson RC, et al. Best practices in intraoperative neuromonitoring in spine deformity surgery: development of an intraoperative checklist to optimize response. Spine Deform. 2014 Sep;2(5):333-9.

2 Diab M, Smith AR, Kuklo TR; Spinal Deformity Study Group. Neural complications in the surgical treatment of adolescent idiopathic scoliosis. Spine. 2007 Nov;32(24):2759-63.
3 Qiu Y, Wang S, Wang B, Yu Y, Zhu F, Zhu Z. Incidence and risk factors of neurological deficits of surgical correction for scoliosis: analysis of 1373 cases at one Chinese institution. Spine. 2008 Mar;33(5):519-26.

4 Pastorelli F, Di Silvestre M, Plasmati R, Michelucci R, Greggi T, Morigi A, et al. The prevention of neural complications in the surgical treatment of scoliosis: the role of the neurophysiological intraoperative monitoring. Eur Spine J. 2011 May;20(S1 Suppl 1):S105-14.
5 Pelosi L, Lamb J, Grevitt M, Mehdian SM, Webb JK, Blumhardt LD. Combined monitoring of motor and somatosensory evoked potentials in orthopaedic spinal surgery. Clin Neurophysiol. 2002 Jul;113(7):1082-91.

6 Nuwer MR, Dawson EG, Carlson LG, Kanim LE, Sherman JE. Somatosensory evoked potential spinal cord monitoring reduces neurologic deficits after scoliosis surgery: results of a large multicenter survey. Electroencephalogr Clin Neurophysiol. 1995 Jan;96(1):6-11.
16

Med Princ Pract 2020;29:6-17

DOI: $10.1159 / 000501256$
Tsirikos/Duckworth/Henderson/ Michaelson 
7 Sutter M, Eggspuehler A, Grob D, Jeszenszky $\mathrm{D}$, Benini A, Porchet F, et al. The diagnostic value of multimodal intraoperative monitoring (MIOM) during spine surgery: a prospective study of 1,017 patients. Eur Spine J. 2007 Nov;16(S2 Suppl 2):S162-70.

8 Sutter M, Eggspuehler A, Muller A, Dvorak J. Multimodal intraoperative monitoring: an overview and proposal of methodology based on 1,017 cases. Eur Spine J. 2007 Nov;16(S2 Suppl 2):S153-61.

9 Quraishi NA, Lewis SJ, Kelleher MO, Sarjeant R, Rampersaud YR, Fehlings MG. Intraoperative multimodality monitoring in adult spinal deformity: analysis of a prospective series of one hundred two cases with independent evaluation. Spine. 2009 Jun;34(14):1504-12.

10 Thuet ED, Winscher JC, Padberg AM, Bridwell KH, Lenke LG, Dobbs MB, et al. Validity and reliability of intraoperative monitoring in pediatric spinal deformity surgery: a $23-$ year experience of 3436 surgical cases. Spine. 2010 Sep;35(20):1880-6.

11 Sutter M, Deletis V, Dvorak J, Eggspuehler A, Grob D, Macdonald D, et al. Current opinions and recommendations on multimodal intraoperative monitoring during spine surgeries. Eur Spine J. 2007 Nov;16(S2 Suppl 2):S232-7.

12 Fehlings MG, Brodke DS, Norvell DC, Dettori JR. The evidence for intraoperative neurophysiological monitoring in spine surgery: does it make a difference? Spine. 2010 Apr; 35(9 Suppl):S37-46.

13 Bhagat S, Durst A, Grover H, Blake J, Lutchman L, Rai AS, et al. An evaluation of multimodal spinal cord monitoring in scoliosis surgery: a single centre experience of 354 operations. Eur Spine J. 2015 Jul;24(7):1399-407.

14 Hamilton DK, Smith JS, Sansur CA, Glassman SD, Ames CP, Berven SH, et al.; Scoliosis Research Society Morbidity and Mortality Committee. Rates of new neurological deficit associated with spine surgery based on 108,419 procedures: a report of the scolio- sis research society morbidity and mortality committee. Spine. 2011 Jul;36(15):1218-28.

15 Nuwer MR, Emerson RG, Galloway G, Legatt AD, Lopez J, Minahan R, et al.; American Association of Neuromuscular and Electrodiagnostic Medicine. Evidence-based guideline update: intraoperative spinal monitoring with somatosensory and transcranial electrical motor evoked potentials. J Clin Neurophysiol. 2012 Feb;29(1):101-8.

16 Nuwer MR, Emerson RG, Galloway G, Legatt AD, Lopez J, Minahan R, et al.; Therapeutics and Technology Assessment Subcommittee of the American Academy of Neurology; American Clinical Neurophysiology Society. Evidence-based guideline update: intraoperative spinal monitoring with somatosensory and transcranial electrical motor evoked potentials: report of the Therapeutics and Technology Assessment Subcommittee of the American Academy of Neurology and the American Clinical Neurophysiology Society. Neurology. 2012 Feb;78(8):585-9.

17 Luk KD, Hu Y, Wong YW, Leong JC. Variability of somatosensory-evoked potentials in different stages of scoliosis surgery. Spine. 1999 Sep;24(17):1799-804.

18 Pelosi L, Stevenson M, Hobbs GJ, Jardine A, Webb JK. Intraoperative motor evoked potentials to transcranial electrical stimulation during two anaesthetic regimens. Clin Neurophysiol. 2001 Jun;112(6): 1076-87.

$19 \mathrm{Ku}$ AS, Hu Y, Irwin MG, Chow B, Gunawardene S, Tan EE, et al. Effect of sevoflurane/ nitrous oxide versus propofol anaesthesia on somatosensory evoked potential monitoring of the spinal cord during surgery to correct scoliosis. Br J Anaesth. 2002 Apr;88(4):502-7.

20 Sloan TB, Heyer EJ. Anesthesia for intraoperative neurophysiologic monitoring of the spinal cord. J Clin Neurophysiol. 2002 Oct; 19(5):430-43.

21 Chen Z. The effects of isoflurane and propofol on intraoperative neurophysiological moni- toring during spinal surgery. $\mathrm{J}$ Clin Monit Comput. 2004 Aug;18(4):303-8

22 Lewis ND, Keshen SG, Lenke LG, Zywiel MG, Skaggs DL, Dear TE, et al. The Deformity Angular Ratio: Does It Correlate With High-Risk Cases for Potential Spinal Cord Monitoring Alerts in Pediatric 3-Column Thoracic Spinal Deformity Corrective Surgery? Spine. 2015 Aug;40(15):E879-85.

23 Leong JJ, Curtis M, Carter E, Cowan J, Lehovsky J. Risk of Neurological Injuries in Spinal Deformity Surgery. Spine. 2016 Jun; 41(12):1022-7.

24 British Society for Clinical Neurophysiology. ANS/BSCN guidelines for neurophysiological recordings of the spinal cord during corrective spinal deformity surgery. 2013. http:// www.bscn.org.uk/content.aspx?Group=guid elines\&Page $=$ guidelines_IOM. 2018.

25 Ahn H, Fehlings MG. Prevention, identification, and treatment of perioperative spi-nal cord injury. Neurosurg Focus. 2008; 25(5):E15.

26 Ziewacz JE, Berven SH, Mummaneni VP, $\mathrm{Tu} \mathrm{TH}$, Akinbo OC, Lyon R, et al. The design, development, and implementation of a checklist for intraoperative neuromonitoring changes. Neurosurg Focus. 2012 Nov; 33(5):E11.

27 Pahys JM, Guille JT, D’Andrea LP, Samdani AF, Beck J, Betz RR. Neurologic injury in the surgical treatment of idiopathic scoliosis: guidelines for assessment and management. J Am Acad Orthop Surg. 2009 Jul;17(7):42634.

28 Jarvis JG, Strantzas S, Lipkus M, Holmes LM, Dear T, Magana S, et al. Responding to neuromonitoring changes in 3-column posterior spinal osteotomies for rigid pediatric spinal deformities. Spine. 2013 Apr;38(8):E493-503.

29 Zuccaro M, Zuccaro J, Samdani AF, Pahys JM, Hwang SW. Intraoperative neuromonitoring alerts in a pediatric deformity center. Neurosurg Focus. 2017 Oct;43(4):E8. 